UVX 2008 (2009) 77-83

(C) EDP Sciences, 2009

DOI: $10.1051 / \mathrm{uvx} / 2009013$

\title{
Spectrométrie VUV par transformation de Fourier à très haute résolution : état et performances du nouvel instrument installé à SOLEIL
}

\author{
D. Joyeux ${ }^{1}$, N. de Oliveira ${ }^{2}$, D. Phalippou ${ }^{1}$, J.-C. Rodier ${ }^{1}$, K. Ito ${ }^{3}$, L. Nahon ${ }^{2}$, \\ M. Vervloet ${ }^{2}$, F. Polack ${ }^{2}$ et M. Roujdane ${ }^{2}$ \\ ${ }^{1}$ Laboratoire Charles Fabry, Institut d'Optique RD 128, 91127 Palaiseau, France \\ ${ }^{2}$ Société civile Synchrotron SOLEIL, Saint-Aubin - BP. 48, 91192 Gif-sur-Yvette Cedex, \\ France \\ ${ }^{3}$ Photon Factory, National Laboratory of High Energy Physics, Tsukuba, Ibaraki 305, Japan
}

\begin{abstract}
Résumé. Un prototype de spectromètre VUV par TF avait été décrit précédemment, en particulier lors de la conférence UVX2004. Ce prototype a été entièrement reconstruit en vue de son installation en ultra-vide sur SOLEIL (ligne DESIRS), avec des spécifications améliorées. Le premier test en VUV a été réalisé en novembre 2007, sur l'argon autour de $79 \mathrm{~nm}(15.7 \mathrm{eV})$. Sur ce premier spectre, le pouvoir résolvant (PR) expérimental obtenu (hors Doppler) est égal au PR théorique, soit 540000 (FWHM) dans les conditions de l'expérience. Depuis, l'instrument a produit les spectres des gaz rares ( $\mathrm{Ne}-\mathrm{Ar}-\mathrm{Kr}-\mathrm{Xe})$, entre 50 et $100 \mathrm{~nm}$, avec un PR (FWHM) supérieur à 700000, en vue d'étudier systématiquement le fonctionnement et ses performances. Nous examinerons particulièrement la résolution spectrale, la précision absolue de l'échelle spectrale, et le rapport signal-bruit. Une chambre échantillon dédiée est en cours de test. Elle comporte une chambre sans fenêtre permettant une pression de plusieurs millibars, et un jet atomique ou moléculaire, pour éliminer l'élargissement Doppler.
\end{abstract}

\section{INTRODUCTION}

Dans le domaine VUV, le meilleur pouvoir résolvant (PR) obtenu à l'aide d'un spectromètre à réseau en spectroscopie d'absorption, est de l'ordre de 200000 (vers $78 \mathrm{~nm}, 15 \mathrm{eV}$ [1]) et n'est guère supérieur à 100000 vers 20 eV $(62 \mathrm{~nm})$ et au delà. Seule la spectrométrie laser a des performances supérieures, mais elle est peu adaptée à l'étude de domaines spectraux larges, et de plus, elle n'accède pas au domaine d'énergie au delà de $20 \mathrm{eV}$. Au contraire, il est clair que la spectrométrie par TF (STF) est en principe susceptible de présenter les performances souhaitées de PR élevé en large bande, à condition de savoir construire l'instrument nécessaire, à savoir un interféromètre à balayage de différence de marche. Or cette réalisation se heurte à un problème technologique majeur: la construction d'une séparatrice large bande, dans le VUV est en effet encore un problème non résolu. Pour cette raison essentielle, aucun spectromètre par TF n'existait au départ de ce travail, un autre projet étant alors en cours de développement [2].

Le laboratoire Charles Fabry de l'Institut d'optique a proposé en 1995 de contourner le problème en utilisant un interféromètre sans séparatrice. Un prototype d'interféromètre à balayage dédié à la STF à haute résolution dans le VUV profond a été réalisé en 2001, permettant d'en valider les principes: il a un pouvoir résolvant (PR) théorique de 500000 à $60 \mathrm{~nm}$ [3]. Ce prototype a fonctionné en 2003 sur source synchrotron, à $195 \mathrm{~nm}$, mais n'a pu être testé dans le VUV.

L'étape suivante, objet de cette communication, a donc été de réaliser un instrument opérationnel sous ultra vide (UHV) propre, en vue de son installation sur la ligne DESIRS de SOLEIL, avec des objectifs de performances améliorées: résolution ultime de $10^{6}$ à $40 \mathrm{~nm}$. Une chambre échantillon dédiée a également été construite; elle comprend une cellule d'absorption sans fenêtre et un jet atomique ou moléculaire polyvalent.

Le nouvel instrument utilisant les mêmes principes que le prototype, [4], nous n'en donnerons qu'un bref résumé. 


\section{PRINCIPES DE RÉALISATION ET DE FONCTIONNEMENT : RAPPEL}

Comme il est indiqué dans l'introduction, l'interféromètre à balayage est du type "à division de front d'onde", ce qui élimine le besoin d'une séparatrice. L'interféromètre est basé sur le prototype "bi-miroir de Fresnel", dans lequel on a remplacé chaque miroir par un réflecteur en toit d'angle $90^{\circ}$. Comme dans le bimiroir de Fresnel, on obtient un champ d'interférence en basculant légèrement un dièdre vers l'autre. Le faisceau incident est "à cheval" sur les deux réflecteurs, ce qui implique qu'ils sont très proches l'un de l'autre. La valeur de l'angle de basculement détermine l'interfrange, et finalement le niveau du signal détecté. L'optimisation de ce paramètre conduit à choisir un angle de basculement de l'ordre de $0.4 \mathrm{mrad}$, d'où une figure de quelques franges, inscrite dans la figure de diffraction de Fresnel produite par les bords des réflecteurs. L'interfrange la plus fine, obtenue à $40 \mathrm{~nm}$, est de $150 \mu \mathrm{m}$, à $130 \mathrm{~cm}$ des réflecteurs.

La qualité principale d'un interféromètre à balayage de différence de marche $(\mathrm{ddm})$ destiné à la spectrométrie par TF est de permettre l'échantillonnage de l'interférogramme à intervalles régulier de $\mathrm{ddm}$. Pour atteindre cet objectif, nous avons adopté un système de motorisation du bras mobile à deux étages. D'une part, une motorisation 1-axe de qualité standard (platine de translation type UMR de Newport) assure un balayage sans à-coup ni vibration (qualité de roulement), mais avec une qualité de trajectoire (guidage angulaire et précision) insuffisante. D'autre part, un dispositif optique original sonde avec une grande sensibilité la position relative des réflecteurs fixe et mobile. Ce dispositif, dont la source est un laser He-Ne monomode stabilisé en fréquence, intègre deux fonctions: 1) un déflectomètre (sensibilité $0.1 \mu \mathrm{rad}$ ) permet de stabiliser la position angulaire du réflecteur mobile par rapport au fixe, via des actionneurs piézoélectriques pilotés par une boucle d'asservissement; 2) un interféromètre multipasse permet de mesurer le déplacement relatif des réflecteurs et donc les variations de la différence de marche au cours du balayage, et de déclencher les acquisitions à des intervalles réguliers de ddm connus et précis, compris entre 20 et $45 \mathrm{~nm}$ (10 valeurs discrètes). Les détails du fonctionnement et de l'implantation peuvent être trouvés en ref. [3] et [4].

\section{DOMAINE SPECTRAL ET RÉSOLUTION : OBJECTIFS DU NOUVEL INSTRUMENT}

Les modifications apportées au nouvel instrument concernent le domaine spectral opérationnel et la résolution.

Le domaine spectral de fonctionnement a été étendu à $\lambda \mathrm{g} 40 \mathrm{~nm}(30 \mathrm{eV})$ au lieu de $60 \mathrm{~nm}$ pour le prototype. Cette extension a été obtenue en remplaçant la couche de platine utilisée précédemment par une couche de SiC. D'autre part, on a intégré dans le dispositif la possibilité d'adapter le pas d'échantillonnage de l'interférogramme en fonction du domaine spectral étudié, par changement du nombre de passages dans le système de contrôle multiréflexion (choix parmi 10 valeurs discrètes, cf. §2). Ceci permet de garantir un PR très élevé sur toute la gamme spectrale accessible, qui va donc de $200 \mathrm{~nm}$ à $40 \mathrm{~nm}\left(50000\right.$ à $250000 \mathrm{~cm}^{-1}$ ou 6 à $\left.30 \mathrm{eV}\right)$.

En ce qui concerne la résolution, l'objectif a été de pouvoir acquérir $1024^{2}$ échantillons au moins entre les différences de marche nulle et maximale, quel que soit le réglage du pas d'échantillonnage. Il s'agit donc d'un interférogramme à balayage asymétrique, qui implique l'utilisation de la transformation de Fourier en cosinus. Dans ces conditions, l'intervalle $\mathrm{d} \sigma$ entre échantillons spectraux est égal à $\sigma_{N} /\left(1024^{2}\right)$, où $\sigma_{N}=1 /(2 \delta)$ est le nombre d'onde correspondant à l'échantillonnage critique, $\delta$ étant l'intervalle de différence de marche entre deux échantillons de l'interférogramme. Une définition de la résolution est la largeur à mi-hauteur (FWHM) de la fonction d'appareil. Celle-ci étant un sinus cardinal on trouve $\mathrm{d} \sigma_{R}=1.2^{*} \mathrm{~d} \sigma$. Le pouvoir résolvant, qui dépend de la région spectrale étudiée, s'en déduit.

La résolution visée guide le dimensionnement du système: la course de balayage et la dimension transversale des blocs optiques doivent être suffisantes pour que le système de contrôle fonctionne pour toutes les valeurs de différence de marche. Compte tenu des éléments mécaniques et de leur implantation, la course réalisable est de $45 \mathrm{~mm}$, et le nombre d'échantillons qu'il est possible d'acquérir 
Tableau 1. Paramètres de réglage et résolution instrumentale théorique pour quatre gaz rares.

\begin{tabular}{|c|c|c|c|c|}
\hline Région spectrale $\mathrm{cm}^{-1}$ & $173000(\mathrm{Ne})$ & $127000(\mathrm{Ar})$ & $115000(\mathrm{Kr})$ & $100000(\mathrm{Xe})$ \\
\hline $\begin{array}{l}\text { Pas d'échantillonnage, } \\
\text { en ddm, }(\mathrm{nm}, @ \mathrm{p})\end{array}$ & $24.49(\mathrm{p}=13)$ & $31.84(\mathrm{p}=10)$ & $35.37(\mathrm{p}=9)$ & $39.80(\mathrm{p}=8)$ \\
\hline $\begin{array}{l}\text { Résolution absolue }\left(\mathrm{cm}^{-1}\right) \\
\Rightarrow \text { Pouvoir résolvant } \\
\text { (FWHM, } 1 \mathrm{~K}^{2} \text { éch.) }\end{array}$ & $\begin{array}{l}0.23 \\
740000\end{array}$ & $\begin{array}{l}0.18 \\
706000\end{array}$ & $\begin{array}{l}0.16 \\
710000\end{array}$ & $\begin{array}{l}0.14 \\
695000\end{array}$ \\
\hline $\begin{array}{l}\text { Résolution absolue }\left(\mathrm{cm}^{-1}\right) \\
\Rightarrow \text { Pouvoir résolvant } \\
\text { (FWHM, balayage maximal) }\end{array}$ & $\begin{array}{l}0.18 \\
940000\end{array}$ & $\begin{array}{l}0.11 \\
1170000\end{array}$ & $\begin{array}{l}0.09 \\
1280000\end{array}$ & $\begin{array}{l}0.076 \\
1320000\end{array}$ \\
\hline
\end{tabular}

dépasse $1024^{2}$ sauf aux plus courtes longueurs d'onde. Il en résulte un accroissement notable de la résolution et du PR, au moins au grandes longueurs d'onde.

Ces relations sont explicitées et illustrées par la table I, qui donne les pas d'échantillonnage adaptés (différence de marche), et les valeurs de la résolution absolue et du pouvoir résolvant associés pour 4 régions spectrales, correspondant à l'étude de quatre gaz rares près de leurs seuils d'ionisation. Ces valeurs ont été calculées dans deux cas: balayage de l'interférogramme pour l'acquisition de $1024^{2}$ échantillons, ou bien pour l'acquisition du nombre maximal d'échantillons permis par l'instrument. Le paramètre $\mathrm{p}$ indiqué est le paramètre de réglage du pas $\delta(\mathrm{ddm})$ d'échantillonnage, avec: $\delta \approx \lambda / 2 \mathrm{p}$, $\lambda=633 \mathrm{~nm}$ étant la longueur d'onde du laser de contrôle.

Enfin, une "voie rouge" (VR) a été insérée dans l'interféromètre VUV. Il s'agit d'une fraction (10\%) du faisceau du laser de contrôle $(633 \mathrm{~nm})$ qui est injectée dans l'interféromètre VUV, parallèlement au faisceau VUV (mais non confondu avec lui). Les interférences (rouges) qui en résultent subissent donc exactement la même loi de différence de marche que les interférences VUV, et peuvent être échantillonnées en même temps que les interférences $V U V$. La raie spectrale qui s'en déduit par TF est alors la réponse instrumentale du spectromètre. Cet interférogramme de référence peut être utilisé de deux manières: il fournit une référence de longueur d'onde, donc une calibration de l'échelle spectrale; il permet également de calculer et en général de corriger les erreurs de position du peigne d'échantillonnage.

\section{DÉTECTION ET PRÉTRAITEMENT DES INTERFÉROGRAMMES}

Les interférences VUV sont détectée à l'aide d'une diode IRD XUV, suivie d'un préampli en courant faible bruit. Les interférences VR sont détectées par une diode visible standard, suivie d'un préampli ad-hoc. Le signal somme (VUV + VR) est ensuite numérisé (16 bits) par une carte PCI, commandée par une horloge externe fabriquée à partir du signal interférométrique de contrôle (sec. 2). Les données sont transférées directement en mémoire du PC.

\subsection{Calcul de l'origine des différences de marche}

L'application de la transformation de Fourier en cosinus requiert que le peigne d'échantillonnage contienne la différence de marche nulle, sous peine d'obtenir une forte distorsion de la fonction d'appareil théorique. En général, ceci n'est pas réalisé, car l'origine n'est connue au mieux qu'à une dizaine de microns près. Il faut donc déterminer l'origine vraie des différences de marche (à $\lambda / 15$ près), puis calculer par interpolation un interférogramme recalé, en utilisant la méthode standard de Forman [5]. A cette fin le balayage est étendu au delà de la différence de marche nulle, de façon à pouvoir effectuer une TF exponentielle à faible résolution. L'étude de la phase dans la bande spectrale d'intérêt permet de calculer précisément l'origine des différences de marche. 


\subsection{Test et correction des erreurs sur l'échantillonnage}

On sait que, en spectrométrie $\mathrm{TF}$, la résolution nominale ne peut être atteinte que si la régularité du peigne d'échantillonnage est suffisante, le critère standard [6] étant: écart aux positions idéales $<\lambda / 15$ (rms, ddm). On peut vérifier à l'aide de la voie rouge que cette condition est réalisée. En effet, celle-ci étant insérée dans l'interféromètre VUV et dans la chaîne de détection, le signal spectral correspondant est disponible sur tout spectre VUV. Comme on l'a dit plus haut, ce signal est la réponse instrumentale du spectromètre. Tout écart à la réponse théorique révèle la présence de défauts, qui peuvent être déterminés quantitativement (par démodulation en phase du spectre convenablement filtré) et corrigés a posteriori, par une interpolation localement constante de l'interférogramme, en utilisant le même algorithme que pour le recalage d'origine. Dans la pratique, on trouve que l'erreur moyenne rms est le plus souvent comprise entre 0.2 à 0.10 fois l'intervalle d'échantillonnage, c'est à dire inférieur à $\lambda_{N} / 10$ à $\lambda_{N} / 20$. Le critère de qualité est donc généralement satisfait sur la plus grande part du spectre. Lorsqu'il ne l'est pas, le processus de correction indiqué ramène le niveau d'erreur à une valeur négligeable, garantissant une fonction d'appareil non distordue.

Dans ce cas, la réponse percussionelle (RP) nominale est un sinus cardinal (sinc), dont la largeur à la base (premiers zéros autour du maximum) est de 2 échantillons spectraux. La largeur à mi-hauteur (FWHM) correspondante est de 1.2 échantillons. L'examen des spectres de raie laser obtenus grâce à la "voie rouge" montre un profil sinc quasi parfait pour ce qui est du lobe principal, et présentant un léger écart avec la théorie dans les premiers lobes. De fait, une telle RP permet d'atteindre la résolution nominale. Toutefois un examen plus attentif de la RP mesurée, en bande plus large et avec une sensibilité accrue, montre la présence de bandes latérales régulièrement espacées, dont les 2 principales sont décalées de $1000 \mathrm{~cm}^{-1}$ et atténuées d'un facteur de l'ordre de 25 par rapport au pic. Ces bandes latérales sont dûes à une modulation parasite de la position du peigne d'échantillonnage due au moteur pas à pas de balayage. En effet, le pas principal de ce moteur est de $5 \mu \mathrm{m}$, soit $10 \mu \mathrm{m}$ en ddm, correspondant au décalage de $1000 \mathrm{~cm}^{-1}$ constaté.

\section{MESURES DANS LE VUV}

Pour évaluer les performances réelles du spectromètre, on a utilisé les spectres des gaz rares. Ce choix est motivé par la simplicité des spectres des gaz monoatomiques par rapport à ceux des molécules, et par le fait que certaines raies (Ar, par exemple) ont été mesurée précisément par spectrométrie laser. La figure 1 (spectre du néon) est donnée à titre d'exemple des résultats obtenus.

\subsection{Résolution spectrale}

Dans les conditions expérimentales utilisées (gaz rares: $\mathrm{He}, \mathrm{Ne}, \mathrm{Ar}, \mathrm{Kr}, \mathrm{Xe}$ à $294^{\circ} \mathrm{K}$, au voisinage de leurs seuils d'ionisation), les raies brutes sont la convolution de la fonction d'appareil avec la fonction d'élargissement Doppler. La largeur Doppler à mi-hauteur (FWHM) est donnée par $\Delta \sigma / \sigma=$ $\sqrt{8 K T \operatorname{Ln}(2) / M c^{2}}=12.310^{-6} / \sqrt{M}$, à $294^{\circ} \mathrm{K}$, (M: masse atomique de l'atome en grammes. Le calcul montre que, sauf pour l'hélium où le Doppler est largement dominant, les largeurs Doppler et instrumentale sont comparables. Il est donc possible de calculer avec une bonne précision la largeur instrumentale réelle à partir de la largeur brute, par soustraction quadratique de la largeur Doppler. Les mesures ont montré un excellent accord avec la largeur théorique pour tous les gaz rares: on retrouve toutes les valeurs théoriques données par la table I pour $1024 \mathrm{~K}$ échantillons. La figure 2 illustre ce résultat dans le cas de l'argon.

Dans le cas de l'hélium, la largeur Doppler est largement prépondérante. Elle est de $1.2 \mathrm{~cm}^{-1}$ alors que la résolution instrumentale peut être de $0.22 \mathrm{~cm}^{-1}$, soit PR $=900000$ pour $\sigma=200000 \mathrm{~cm}^{-1}$. Ceci illustre la nécessité d'un "environnement échantillon" dédié à la très haute résolution, et permettant l'élimination du Doppler (refroidissement par jet). La nécessité de refroidir apparaît également dans 


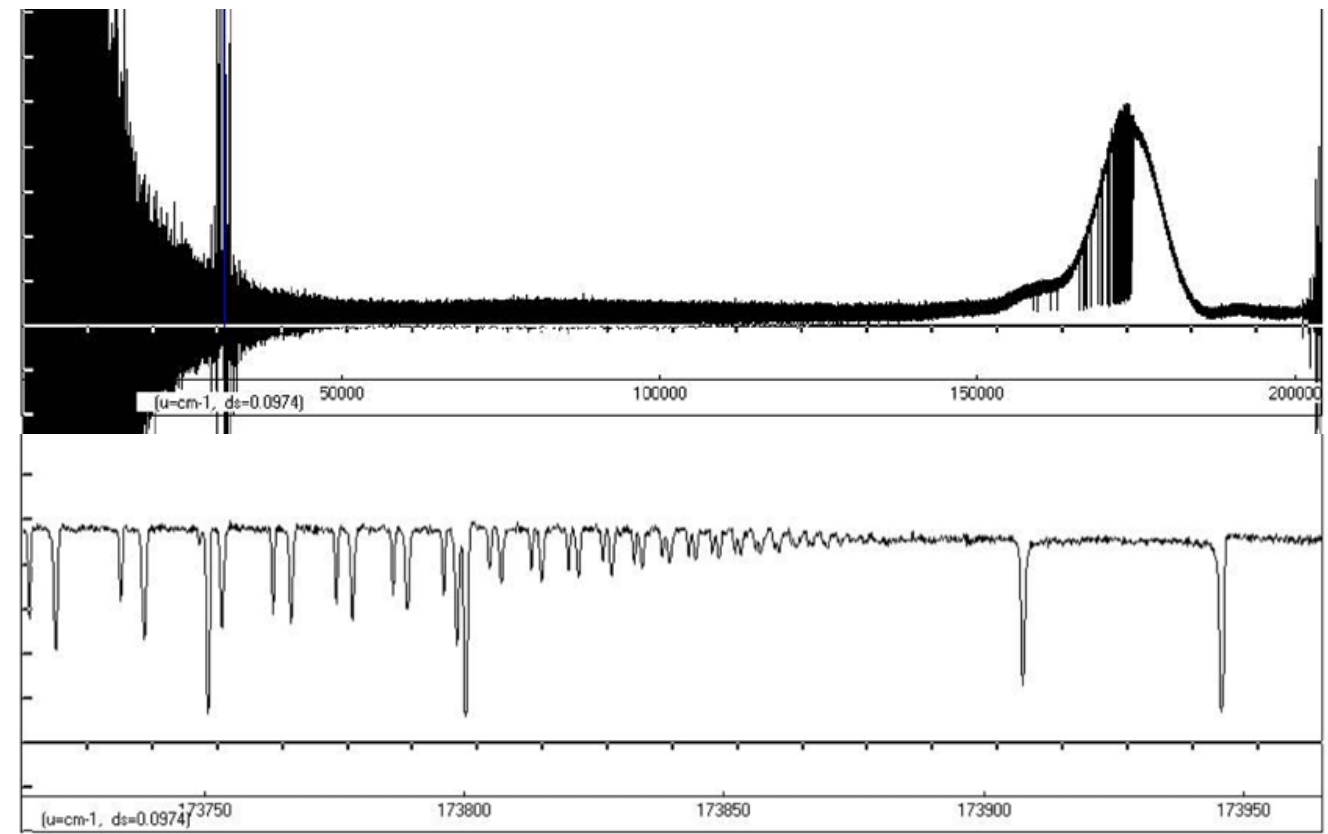

Figure 1. Spectre du néon obtenu par moyenne des spectres issus de 86 balayages. En haut: spectre total de $\sigma=0$ à $\sigma=205000 \mathrm{~cm}^{-1}$. En bas: bande de $250 \mathrm{~cm}^{-1}$ autour de $173800 \mathrm{~cm}^{-1}$. La résolution instrumentale (FWHM) de $0.234 \mathrm{~cm}^{-1}$ résulte de l'acquisition de $1024 \mathrm{~K}$ échantillons, espacés de $24.49 \mathrm{~nm}(\mathrm{ddm})$, cet intervalle étant connu avec une précision relative meilleure que $10^{-6}$.

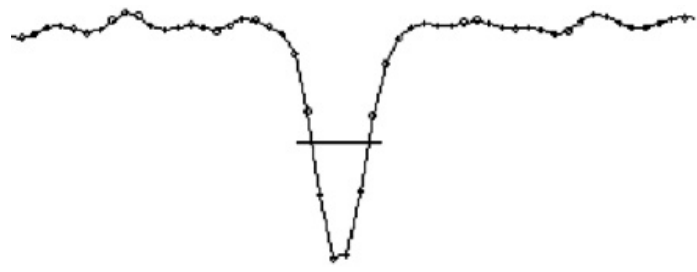

Figure 2. Raie non saturée de l'argon à $126650.81 \mathrm{~cm}^{-1}$. La largeur mesurée est de $0.31 \mathrm{~cm}^{-1}$, FWHM. La largeur Doppler calculée à $21^{\circ} \mathrm{C}$ pour l'argon est de $0.246 \mathrm{~cm}^{-1}$. On en déduit une largeur instrumentale calculée par différence quadratique de $0.189 \mathrm{~cm}^{-1}$, pour une valeur théorique de $0.180 \mathrm{~cm} E^{-1}$. Les mesures effectuées avec les autres gaz rares (hors hélium, cf. texte) ont montré le même accord. (Profil de raie interpolé $\mathrm{x} 4$ ).

l'étude d'espèces moléculaires, en particulier d'intérêt astrophysique, car l'analyse des bandes de rotation-vibration devrait être fortement améliorée par une telle augmentation de la résolution spectrale. La chambre dédiée à la STF permet d'ores et déjà une pression de quelques millibars sur une dizaines de centimètres dans la cellule ouverte. Le jet moléculaire est en cours de mise au point.

\subsection{Bruit dans les spectres mesurés}

Comme le montre la figure 1, l'ensemble du domaine spectral calculé est sujet à au moins deux types de bruit. Aux faibles valeurs du nombre d'onde ("basse fréquence") on constate une forte montée de bruit. Celle-ci est essentiellement due à toutes les perturbations d'origine mécanique: vibrations transmises par 
le sol ou par la ligne, mais également déplacements parasites du faisceau par rapport à l'interféromètre. En général ces perturbations sont limitées dans le spectre aux régions de faible nombre d'onde. En ce qui concerne la partie VUV, nous avons vérifié que le bruit spectral est principalement attribuable au bruit de photon.

La précision sur les mesures d'absorption est limitée par le rapport signal/bruit dans le spectre, mais également par deux autres facteurs: la précision sur la connaissance de la densité d'échantillons, et la détermination du spectre de référence (spectre à densité nulle, dits "blanc"). En ce qui concerne la première, sa précision est limitée, comme pour tout système du même type à cellule ouverte ou à jet, et il peut être nécessaire d'étalonner la densité de colonne à l'aide de raies dont la section efficace est connue. Pour ce qui est de la précision sur les spectres blancs, elle est affectée par des instabilités spectrales apparentes liées à des instabilités du faisceau VUV par rapport au spectromètre. L'amélioration des performances sur ce point passe par l'utilisation de données a-priori (quand elles existent) et/ou par l'amélioration de la stabilité VUV/instrument, ainsi que l'acccumulation statistiques de spectres blancs.

\subsection{Reproductibilité et précision absolue de l'échelle spectrale}

Bien que le pas d'échantillonnage des interférogrammes soit basé sur une raie laser He-Ne stabilisée en fréquence (stabilité à moyen terme $10^{-8}$ ), la reproductibilité comme la précision de l'échelle spectrale sont limitées par la stabilité et la connaissance d'éléments géométriques. Certains éléments (angles entre les différents réflecteurs) relèvent de la fabrication et de mesures optiques, et sont très bien connus, alors que d'autres relèvent d'éléments mécaniques (vecteur translation du bras mobile) ou du positionnement des faisceaux laser et VUV, qu'il est difficile de régler par rapport aux éléments mécaniques à beaucoup mieux que $1 \mathrm{mrad}$. De ce fait la précision relative intrinsèque (sans utilisation de référence interne ou externe) est de l'ordre de $10^{-6}$. Cette valeur peut descendre à 2-3 $10^{-7}$ si l'on utilise le faisceau VR comme marqueur de fréquence. De même, la stabilité relative intrinsèque est limitée par la stabilité angulaire du faisceau laser de contrôle et du faisceau VUV. La dérive spectrale qui en résulte n'est pas supérieure à 1-2 $10^{-7}$ à l'intérieur d'une série d'acquisitions (en vue d'obtenir un spectre moyen, par exemple). Elle peut être supérieure d'un ordre de grandeur d'un jour à l'autre, après injection d'un nouveau faisceau synchrotron et/ou extinction/allumage du laser de contrôle, à moins de procéder un réglage soigneux de l'instrument.

\section{CONCLUSION}

Le nouveau spectromètre VUV par TF installé sur la ligne DESIRS de SOLEIL a démontré des performances de résolution uniques, qui dépassent les objectifs fixés au départ. Le rapport signal/bruit spectral est limité par le flux disponible. Le bruit prépondérant étant le bruit de photon, l'amélioration du s/b spectral nécessite l'accumulation de spectres indépendants, ainsi que le choix d'un compromis sur la résolution. En ce qui concerne la fidélité et la précision de l'échelle spectrale, elles sont toutes deux nettement meilleures que $10^{-6}$.

En l'état actuel, cet instrument à ultra-haute résolution a des performances démontrées uniques au monde. Il a vocation à être un outil de spectrométrie ouvert à la communauté des physico-chimistes de la phase diluée, intéressés par l'étude des niveaux électroniques excités et super-excités des atomes, des molécules et des radicaux.

La réalisation de l'instrument installé sur la ligne DESIRS du synchrotron SOLEIL a été financée par le contrat ANR-05-BLAN-0364. Elle a été menée à bien grâce au travail et au savoir-faire hautde-gamme des membres du groupe surfaces et composants optiques du Laboratoire Charles Fabry de l'Institut d'Optique (polissage, assemblage, et métrologie du “cœur optique” de l'instrument). 


\section{Références}

[1] L. Nahon, C. Alcaraz, J.L. Marlats, B. Lagarde, F. Polack, R. Thissen., D. Lepere, K. Ito, Rev. Sci. Instrum. 72, (2001) 1320.

[2] M.R. Howells, K. Frank, Z. Hussain, E.J. Moler, T. Reich, D. Moller, D.A. Shirley, Nucl. Instrum. Meth. A347,(1994) 182.

[3] Nelson De Oliveira, “ Etude et réalisation d'un spectromètre par transformation de Fourier, sans lame séparatrice, pour le domaine spectral VUV-EUV ”, PHD de l'Université Pierre et Marie Curie (Paris VI), mai 2001.

[4] D. Joyeux, N. de Oliveira, D. Phalippou, J. C. Rodier, M. Vervloët, F. Polack, L. Nahon, J. Phys. IV France 127, (2005) 77-85.

[5] M.L.Forman, W.H. Steel and G.A. Vanasse, J. Opt. Soc. Am. 56, (1966) 59.

[6] J. Chamberlain, "The principles of interferometric spectroscopy”, (J. Wiley, New York, 1979) 